\title{
How Do Designers Feel Textiles?
}

\author{
Bruna Petreca, Sharon Baurely \\ Design Products \\ Royal College of Art \\ London, UK \\ bruna.petreca@network.rca.ac.uk, \\ sharon.baurley@rca.ac.uk
}

\author{
Nadia Bianchi-Berthouze \\ UCLIC \\ University College London \\ London, UK \\ n.berthouze@ucl.ac.uk
}

\begin{abstract}
Studying tactile experience is important and timely, considering how this channel is being harnessed both in terms of human interaction and for technological developments that rely on it to enhance experience of products and services. Research into tactile experience to date is present mostly within the social context, but there are not many studies of the understanding of tactile experience in interaction with objects. In this paper, we use textiles as a case study to investigate how we can get people to talk about this experience, and to understand what may be important to consider when designing technology to support it. We present a qualitative exploratory study using the "Elicitation Interview" method to obtain a first-person verbal description of experiential processes. We conducted an initial study with 6 experienced professionals from the fashion and textiles area. The analysis revealed that there are two types of touch behaviour in experiencing textiles, active and passive, which happen through 'Active hand', 'Passive body' and 'Active tool-hand'. They can occur in any order, and with different degrees of importance and frequency in the 3 tactile-based phases of the textile selection process - Situating, Simulating and Stimulating - and the interaction has different modes in each. We discuss these emerging themes in the textile touch process, to inform the design of technology to support it, and to take the textile touching experience to further understand aspects of affective touch beyond social touch.
\end{abstract}

Keywords-tactile experience; touch behaviour; textiles; designers; Elicitation Interview

\section{INTRODUCTION}

Studying tactile experience is important and timely, considering how this channel is being harnessed both in terms of human interaction and for technology developments that rely on this channel to enhance experience of products and services. Research on the tactile experience to date is present mostly within the social context - both in direct human affective touch [1] and mediated through technology [2][3] -, but there are not many studies on the tactile experience in interaction with objects [4]. However, "Our relationship with things is not a distant one: each speaks to our body and to the way we live" [5]. This brings to the surface a crucial question on the relation between materials and tactile experience, particularly on how the body plays a role in this interaction.

In this paper we use textiles as a case study to investigate how we can get people to talk about this experience, and to understand what may be important to consider when designing technology to support it. We focus on experiences with textiles, these materials that invariably come into close contact with our skin [6], and surround us in diverse applications. In the textile realm, online trade, digital co-design and materials selection are becoming part of the designer experience when designing, purchasing and commercialising products. However the digital tactile channel is impoverished or in most cases not available, despite its importance for consumers [7] and designers [8]. The textile industry provides methods to describe and measure what is called 'textile hand' - these are the sensations and impressions resulting from interaction with textiles [9]. Unfortunately, these methods are very technical, focusing on usability aspects and therefore not able to capture or take into account the tactile experience of the designer. This gap in current research is reported as a need to support the balance between technical and experiential information beyond textiles, but broadly in materials and design research [10][11].

Previous research attempted to create technology that supports the experience with textiles through their digital representation, by exploring tactile [12][13] or other sensory channels (such as sound [14]). Whilst these are making progress in overcoming technological limitations, they show and adopt a narrow understanding of experiencing textiles. This is highlighted, for example, by designers' feedback when engaging with such type technology, reporting that they need to feel [15] - where feel is related to movement, surface texture, but also to the engagement with the physicality of a fabric and all other touch behaviour types that it affords beyond hand manipulation. Here we report a study conducted to explore a key question: how do designers experience textiles when they select them within their design process? We conducted a qualitative exploratory study through a first-person approach, using the 'Elicitation Interview Method', in which 6 experienced professionals from the fashion and textiles area were interviewed. Through this study we aim to contribute in two ways: (1) to further our understanding of textile touch process, to inform the design of technology to support it, and (2) to take the textile touching experience as a way to further understand aspects of affective touch beyond social touch.

\section{BACKGROUND RESEARCH}

\section{A. Distinct Approaches to Tactile Experience with Textiles}

There is an on-going unbalance on how the tactile experience with textiles is dealt with between industry segments. While the textile engineering research is populated by objective approaches, designers mostly rely on tacit and 
implicit knowledge to inform decisions. To investigate the attributes that affect the perception of the 'textile hand', the textile industry employs specific objective measurements. The most significant attempt towards standardising evaluations was made by the 'Hand Evaluation Standards Committee' (HESC), convened in the 1980s by Kawabata to create a set terminology for the 'subjective evaluation' of hand [16]. The HESC created both the most often cited definitions (although they are reported as problematic to translate to other languages) and the most usually acknowledged system of objective hand measurement, the Kawabata Evaluation System of Fabric. The latter has been applied within the textile manufacturing industry for allowing consistent analysis, i.e. fabrics are judged on predefined scales (i.e. compressional, tensile, shearing and bending properties). Their main focus is in identifying the characteristics and behaviour of textiles, while no mention is made to how the experience emerges and how the tactile behaviour contributes to forming it. In addition, the tactile experience used in these tests is limited to mechanical dimensions, and affective dimensions remain unarticulated [17]. In fact, the engagements of designers with materials rely on knowledge that is innate (reliant on designers' sensibility and intuition) and tacit (acquired through training and experience) [8], which remain underexplored within the textile realm and fail to inform the design of technology.

\section{B. Missing Textile Feel}

Design tools and sourcing activities are increasingly going digital, but interactive technology for textiles and fashion are still very limited mainly to visual and verbal channels, with few initiatives exploring haptic feedback [18][19]. Although handling a textile is crucial for its appreciation and understanding [17], current technology is still unable to support this rich experience.

Previous studies attempted to tap into our subjective relationship with textiles, physically and digitally, and the research methods applied were useful for developing understanding on how to represent these subjective aspects of the interaction [13] and to devise new technology and methods for eliciting and transmitting perceptions of tactile qualities of textiles [20]. One of the outcomes produced were the interactive videos for the application 'iShoogle' [21] - an interface that enables interactive simulation of digital textile handling for a touch-based display. The interactive videos (iShoogle) were inspired by phenomenology, as it included the experience of actively investigating the textile through movement and related visual feedback [22]. Whilst the resulting interactive videos represented a clear step forward from current stills, as it introduced engagement through active manipulation of the textile as well as a better understanding of some properties, these were considered to provide an impoverished experience by fashion and textiles professionals because they reported that they could not feel [15]. Such feedback led us to question in this paper what is 'feel' for designers, or rather, how do designers feel? Here we pursue more detailed descriptions to access dimensions of the experiences resulting from interactions with textiles that remain unarticulated or even unconscious.

\section{THE STUDY}

To explore the above question, in this study we have chosen a specific context of inquiry where tactile interaction with textile is central: how do designers experience textiles when they select them within their design process? We are interested in understanding the tactile interaction, its experience and its forms during the selection process. 6 experienced professionals from the fashion and textiles area were interviewed. Their profile is provided in Table I.

\section{A. Elicitation Interview Method}

We follow a qualitative approach based on the 'Elicitation Interview Method' [23], which combines psychology and phenomenology approaches [24] to obtain a first-person [25] verbal description of cognitive and experiential processes. This method aims at assisting interviewees to verbally explain the subjective experience that is generally inaccessible, unknown or difficult to articulate. The focus of these interviews is in the "how" and "what" of the experience, while "why" is never asked [24]. This is to avoid explanations or abstract considerations and focus in revealing introspective acts "through specific prompts and questions, in order to help a subject to become aware of the unrecognized part of the process being described" [23]. Such questions are content empty (not inducing), and instead explore the unfolding of the experience in time ('Diachronic' dimension) and the facets of an experience in a specific time ('Synchronic' dimension).

\section{B. Procedure}

The participants were met individually and a short interview was conducted to demonstrate the method. In all cases they agreed to be interviewed, and a consent form was signed, which was approved by the local ethics committee. The interview consisted of two phases: (Phase 1) Designers were given a textile sample of $(20 \times 20) \mathrm{cm}$, which they were invited to explore, and right after they were interviewed about their tactile experience with that specific textile. This interview lasted for a maximum of 20 minutes. (Phase 2) Designers where interviewed about their experience when selecting textiles. The experience could be either from a previous project they have worked on, or they were given the option to make a selection on spot, just before the interview - for this a set of textile samples and a design brief were prepared to allow an in situ approach [12][26].

TABLE I. PARTICIPANTS PROFILE

\begin{tabular}{|c|l|c|l|}
\hline ID & \multicolumn{1}{|c|}{ Field of experience } & Years & Gender \\
\hline Fashion 1 & Womenswear couture and teaching & 20 & Female \\
\hline Fashion 2 & Womenswear high-street & 7 & Female \\
\hline Fashion 3 & $\begin{array}{l}\text { Menswear high-street, couture, } \\
\text { teaching }\end{array}$ & 30 & Male \\
\hline Costume 1 & Costume design for television & 20 & Female \\
\hline Textile 1 & Textile designer and researcher & 7 & Female \\
\hline Textile 2 & Textile researcher & 10 & Female \\
\hline
\end{tabular}


The interviews lasted for 40 minutes maximum. Both interviews were video recorded when there was consent from the participant. The interviewer had received a total of 3 weeks training on the 'Elicitation Interview Method' by an expert over the previous two years.

\section{Analysis}

All data was transcribed for analysis, including annotations from observing the videos, using the Thematic Analysis method, following Braun and Clarke's [27] guide- lines. Coding was conducted using QSR International's NVivo 10 software. Our question was used to guide the analysis, but focus was given to themes that emerged from responses, which are described in the results section. We first conducted a Thematic Analysis to identify the most important themes. This was to produce a high-level picture of the tactile experience by designers, particularly in terms of their behaviour (the focus of this paper), before delving into details of the dynamics of this experience. To address the latter, the analysis identifies the 'Synchronic' and 'Diachronic' dimensions of the experience, which will be reported in a separate paper.

\section{DESIGNERs TACTILE EXPERIENCE WITH TEXTILES}

Three main tactile-related themes emerged from the analysis: 'Situate', 'Simulate', and 'Stimulate'. In addition, the analysis of verbal reports combined with the video-recordings show different types of attention and touch disposition according to the type of engagement with the textiles, that can be categorized mainly as: 'Active hand', i.e. touching to explore the textile, to perceive the tactile experience and to use it to interpret the feeling from the textile; 'Passive body' (generally not the hand), i.e. being touched, only receiving, without the possibility to actively change how the fabric will behave on it; and 'Active tool-hand', i.e. as an enactive tool, a driver of experience, through shaping how the textile is going to interact - the if, when, how and what the textile will touch with the aim not to experience it but to let another agent to experience it (e.g., our own body or someone else body). The themes and the role of active and passive touch within these themes are discussed below.

\section{A. Situate}

When designers encounter textiles they situate, i.e. all touch behaviours observed in these moments are about understanding the textile and how it feels to the respondent. The touch behaviour aims at feeling it through the different types of receptors: first, through the discriminatory ones, mainly from the hand, to understand what it is, and then through the affective receptors [28] (not on the hand) to feel the sensation it produces.

Fashion 1: "I was able to hold it and understand it and use my knowledge, I was like, "Okay, I think I know what it is". (...) Once I knew what it was, I put it next to my skin but not in my hand."

These behaviours are further described below.

1) Understanding the textile with hand

Here the touch behaviour is mostly 'Active hand' centred, i.e. using one or both hands, in diverse ways - these are the closest to the touch behaviours explored by textile engineering research as surveyed in [17]. Their touch behaviour aims to explore and compare characteristics of the textiles. They touch it to experience its properties and how the textile reacts to their touch. Designers focus on the physicality of materials, and probe them to enable understanding.

Fashion 1: "Is this what I want? Is this the right thickness? What's it feel like, and what's it's bias? Like, does it stretch? Just what are it's-- what it's quantity, if you like. "Okay, it could be a twill silk. It could be a cotton."

Designers performed diverse touch behaviour according to the property they were investigating, e.g. hold by the corner to see how it falls (drape), squeeze and/or drop to feel weight (e.g. in the extract below), shake or stroke to feel temperature (warm, cool, fresh, and so on), pull to feel its resistance (weak or strong), touch around the edges to understand size (Fig. 1).

Interviewer: And how does it feel when it feels light?

Fashion 2: Ah... it feels airy like [moving hands to show the feeling]... like it can fly a bit and go...

Interviewer: Hum-hum. And what do you do to feel that it is light?

Fashion 2: Also just by squeezing and dropping.

This understanding is also related to how they touch the textiles to anchor [29] their thinking about how they would behave in the making, e.g. in both extracts below.

Costume 1: Because the thickness has to be right, the weight, the way that it goes along your fingers, the way that it bends. The thickness, so that you know that it is not going to be very bulky when you've sewn it.

Interviewer: And when you imagine that you are sewing it, how do you do that?

Fashion 2: Ah it is also the same... also by stretching it and seeing how consistent is the fabric and how it will slide [moving hand as if she was inserting the fabric] through the machine.

\section{2) Understanding textiles on me}

Here the touch behaviour is a combination of 'Active hand', 'Passive body' and 'Active tool-hand'. Designers used one of their hands actively to touch another body part, usually the hairy skin on the forearm. Through this orchestration, they were able to feel how it is to be touched by that textile. All the interviewees did this only after they thought they knew the textile properties and wanted to explore their own bodily reaction to it. In some cases, the designers referred to the textiles as 'living' things, which 'want' (Fashion 1 quote below and Fig. 2) and 'move' (Fashion 2 quote below) by themselves.

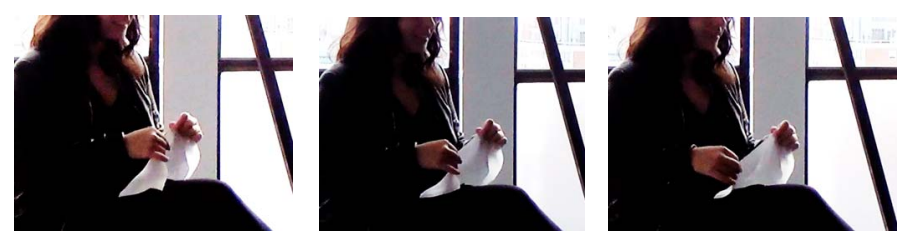

Fig. 1. Fashion 2 touching edges to understand the size of the textile swatch.

Fashion 1: Yeah. To me it's all about, "What does that feel like? Do I want it close to my skin? Will it hold a tighter fit? Does it want to be a little bit looser?" 

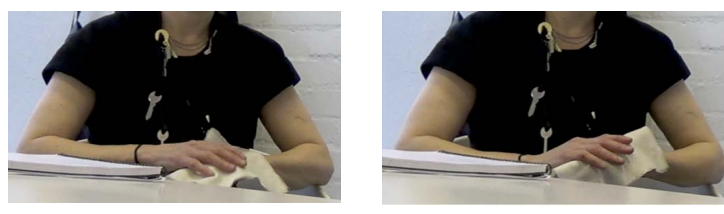

Fig. 2. Fashion 1 active and passive touch.

Interviewer: So when you feel with other parts of the hand, what does it feel like? How do you do that?

Fashion 2: Just by putting it on the top of my hand and moving it. Interviewer: And then what happens next?

Fashion 2: It is very fast. You just rub it very fast in your skin, and then you feel how it moves, and how it falls. (...) Because when you touch with the fingers you get pressure, and then you get pressure from feeling the fingers again. And when you put the fabric on top of your hand, then is just the fabric.

There is a continuous shift between the body surface that experiences and the 'Active tool-hand' that is there to drive the experience of the other body part rather than its own.

\section{B. Simulate}

After understanding the textile, its behaviour and one's body reaction to it, the designers use tactile exploration to simulate concepts and support the creative process. To aid selecting the most suitable textile to a design brief, the body is used as a surface to test ideas, e.g. where on the body we are going to wear the textile. Here again, there is an orchestration of active and passive touch, which involves mostly 'Passive body' and 'Active tool-hand' types. This time however, it is not about feeling the textile, it is rather about feeling the concept that is made by the textile.

Fashion 1: Most fabrics I would generally have put up against my body. And because this has big holes, I have held it against my skin or on my chest over my bra, because it's the kind of fabric is, "Oh, do we need to make an under piece?

The 'Active tool-hand' was used to produce different shapes, drapes or folds with the fabrics, and then these were placed using the other arm or other body parts as support 'Passive body'. Both the active touch that manipulates and the passive touch that receives the results of the manipulation participate and support the process of forming the design concept (see Fig. 3 for an example of a sleeve simulation by Fashion 1). Here again, the 'Active tool-hand' seems to become a facilitator and a driver for the projection of the textile as an animated thing. Because fashion designers create for bodies in movement, this combination of movement, feeling and visualisation over the body supports imagination in a very implicit manner. This is also used to determine the best way to apply the textile to a design. The 'Active toolhand' directs the 'if, when, where (what part of the body) and how' (its weight, speed, direction, texture) the textile-based concept (e.g., a simulated sleeve) will touch the body.
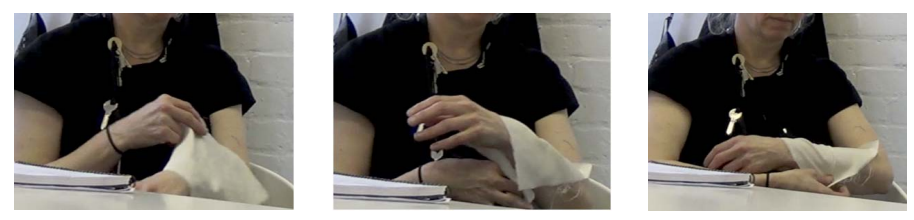

Fig. 3. Fashion 1 simulating a sleeve.
While this is happening, the body experiences manipulation through an alternation of contact and the lack of contact with the textile. The tactile experience is blocked from the 'Active tool-hand', as it is active in driving the experience of another part of the body that receives the textile. There seem to be a continuous switch in the attention from the tactile perception in the hand to the tactile experience in the body, and the attention focus is on the part of the body that is being touched. The 'Active tool-hand' seems to become part of the simulation that the designer is projecting, in a similar manner to what Kirsh [29] observed with dancers when they use their hands in a sketching manner to practice dance sequences.

\section{Stimulate}

Stimulation is about experimenting beyond the initial concept, by stimulating an experience that the textile can provide. This experience helps designers in building metaphors. Using diverse touch behaviours and experiences that derive from them, designers manipulate textiles to enable associations. These are mostly active and use both hands, but sometimes they involve other parts of the body. Here designers seem to explore the relation between sensation and metaphor in ways that lead to very different types of touch behaviour than have been previously reported in the textile engineering literature (for a review see, [17]). Metaphor use is crucial for the design process as a communication tool. The metaphor generates the gesture and the tactile experience that emerges will reinforce, or complete, or refine the metaphor. There is here a relation between touching, thinking, and imagining, which will be discussed later in light of Clark's [30] propositions. When 'stimulating', designers create verbal and visual metaphors based on their movements and the textile reactions, which are described in 1 and 2 below.

\section{1) Feeling moves}

From the interviews it is highlighted that what moves these professionals is to sense that this physical encounter presents new possibilities. The touch behaviour and the movement of the textiles afford multiple opportunities for transformations. The designers move to find new interactions and to experiment with new ways of being stimulated. The textile is present as an animator (in the epistemology of the word, as something that animates, gives motion, inspire) of bodily configurations that on their own would seem impossible to be lived or communicated, as they rely on the textile's reaction. This is the case of the feeling of "feathers and air" (Fashion 1), or "butterflies" (Costume 1), or "fresh and not cheap" (Fashion 2 ). These are verbal indicators of the affective tactile experience taking a more important role, and here the touch behaviour observed is unconstrained. It is this imaginative (or, in their words, "wondrous") relation with fabrics that inspires these experts, and indicates this perception of textiles as 'alive'. The touch behaviour here is playful, free and dynamic. Taking the metaphor "feathers and air" as an example (Fig. 4), the designer moved her hands in front of the body, facing each other, in very rapid circular movements, having the textile loose and floating between the hands. The fabric falling in her hands would trigger her movements to then throwing it up again. 

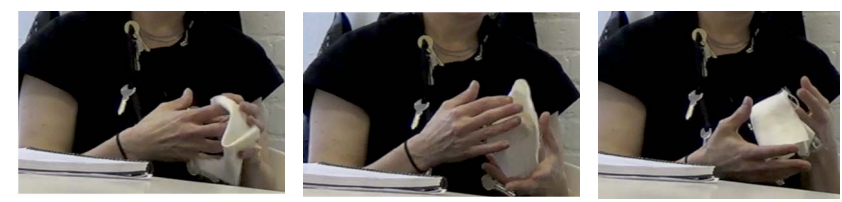

Fig. 4. Fashion 1 stimulating for "feathers and air".

\section{2) Touch enabling tactile mental imagery}

Through touching the designers visualise the textile, i.e. a textile is imagined, based on its perceived tactile characteristics. This is helped by movement, which allows them to experiment new ways of stimulation. As suggested earlier by Kirsh [29] on imagination, when interviewees imagine the fabrics they create a visual experience.

Textile 1: I can see the textile image through my hands. I can see it much thicker than I know it actually is. But at the moment I hold it in my hands, I could see it much thicker.

\section{DISCUSSION}

\section{A. Modes of Feeling}

Designers are feeling their way in interactions with textiles. These are sensuous, affective encounters. Clark [30] discusses brains as predictive machines, and suggests that perceiving, knowing and imagining are intrinsically related activities, which seems to connect to our identified themes and their dynamics. Our study indicates that the manner in which designers experience textiles is crucially related to touch behaviour and bodily engagement; the hand is at time the one that experience the touch and at time the one that drive the experience on a different body part. These touch behaviours involve both active and passive touch, in different intensities and modes, within the three main behaviours we identified as Situate, Simulate and Stimulate.

This relates to the literature in cognition and dance studies, which suggests that we move to experiment new ways of stimulating [31]. As proposed by Nöe [31], perception and movement are intrinsically dependent - we enact our feelings (perceptual experience). In the case of designers, when 'Situating' they enact their perception, they move to understand; both when 'Simulating' and 'Stimulating', designers manipulate textiles to experiment diverse stimulations while imagining certain pieces of clothing, and experiences they evoke. The 'Active hand', the 'Active-tool hand' and the 'Passive body' are there to enact the experience and to form the knowledge of the fabric, how the body will react to it and what the fabric may become. Only through acting on a fabric certain qualities of the fabric are revealed. In our study, variations on touch behaviour through which designers interact with fabrics enables them to discover more about the fabrics, to imagine possible applications, and to go beyond concepts by stimulating diverse experiences that the textile can provide.

\section{B. Beyond Regular Tactile Behaviour}

The literature of affective touch has revealed a relationship between the emotion conveyed and the type of touch and its kinematics [1]. With our study, we encountered other important aspects of the tactile experience: a) movement that enable diverse interaction types and experiences, as well as alter the textile that reacts and provides different stimulations; b) and attention shift (which is related to the touch behaviour types). Specifically, in 'Simulate' the 'Active tool-hand' is shaping the surface that will touch the body, and shaping the dynamic of the surface as it touches the body to dynamically create the tactile experience. By going beyond Kawabata's method [16], mainly aimed at eliciting a verbal description of fabric properties or expressing it numerically, we uncover a more complex process of experiencing textile through touch.

The elicitation method used in our study had been previously applied in the HCI context [12][26] to help people to talk about their subjective experience of different products (e.g., food [32]). We went one step forward here, proposing not only to understand what people feel, but also to understand how the tactile process take place. By bringing to light more facets of the process and the role of interacting parties, and not just the discrimination of the final experience, we better understand what technology needs to provide to recreate that process when the channel is missing, or when an artificial agent needs to experience it. In our research, we aim to create technology that enhances the important elements of the tactile experience during the selection of textiles. In one of our studies, after experiencing tools that captures and displays visually aspects of tactile behaviour (e.g., the amount and timing of finger pressure on fabric) [34] designers suggested that such information could help them better understand their experience and not just the material qualities. They considered such technology could help re-live the experience when back in their studios, where the hundreds of textiles experienced at a fair were available only as pictures or videos [35].

\section{Supporting Freer Manipulation for Exploration}

In the descriptive touch, mainly in 'Situate', free manipulation is key for investigating the characteristics of the fabrics. This is not supported by current touchscreens, as previously discussed [17]. Freer manipulation is key for feel, which for designers is related to a free sensorimotor experience that entails understanding what the fabric is and imagining what it could become. With most of the technology that is currently available the focus is located in mimicking textiles properties, but not in supporting or enhancing interactions. Taking Simulate or Stimulate themes as examples, the touch behaviours identified go much beyond feeling the textile physical properties, but into what designers can make with it, and how they are affected by moving it freely with hands and other body parts.

\section{Supporting Full Body Engagement and Not Just the Fingers}

The body is crucial for supporting understanding and to project (as suggested in [29]) while imagining what the textile could become when manufactured, as well as being involved in experimenting new experiences (i.e. new ways of getting stimulation). Research around technology has also shown that natural body movement [22] and touch behaviour [36] improve the emotional engagement with the task and affect the perception of what is evaluated. Hence, supporting full body 
engagement when designing technology could be used: (1) to provide information or a better experience when communicating characteristics of textiles digitally, (2) to provide multisensory feedback on the body when manipulating textiles, which would augment this experience and could enrich even more moments like in Stimulation to increase the possibility of metaphors generation.

\section{CONCLUSION}

Participant's contribution revealed that there are two types of touch behaviour in experiencing textiles, active and passive, which happen through 'Active hand', 'Passive body' and 'Active tool-hand'. They occur in any order, and with different importance and frequency in the 3 tactile-based phases of the selection process - Situating, Simulating and Stimulating - and the interaction has different modes in each. During 'Situating', the tactile behaviours are the specialist ones to understand the textile, similar to the discussed by the textile engineering literature, and which designers develop and specialise through training and practice to interrogate textiles. As the textile is understood, the affective tactile experience starts to take a more important role and the tactile behaviour is unconstrained. It is about understanding the textile through and on the body - what it is and how it feels. 'Simulating' is about creating concepts through tactile exploration and 'Stimulating' is about experimenting beyond the initial concept through tactile exploration to generate metaphors. The hand is at time experiencing and at time an active tool that drives the tactile experience of a different body part. These are relevant behaviours to tactile experiences with textiles that we have not accessed with previous methods, and no previous descriptions were encountered in the fashion and textile literature, or more generally in relation to materials experience.

\section{REFERENCES}

[1] M.J. Hertenstein, R. Holmes, M. McCullough, D. Keltner, The communication of emotion via touch. American Psychological Association, 9(4), 2009, pp.566-573.

[2] G. Huisman \& A. Darriba Frederiks, Towards Tactile Expressions of Emotion Through Mediated Touch. CHI 2013, 1575-1580.

[3] Y. Park, K. Baek, T. Nam, "The Roles of Touch during Phone Conversations: Long-Distance Couples' Use of POKE in Their Homes,". Proceedings CHI 2013.

[4] N. Bianchi-Berthouze \& A. Tajadura Jimenez, It's not just what we touch but also how we touch it, 2014.

[5] M. Merleau-Ponty, Phenomenology of perception. New York: Routledge, 2012.

[6] A. Gallace \& C. Spence. In touch with the future. United Kingdom: Oxford University Press, 2011.

[7] A. Citrin, D. Stem, E. Spangenberg, M. Clark, Consumer need for tactile input: an internet retailing challenge. Journal of Business Research, 2010, 56, 11, 915-22.

[8] P. Dormer, The culture of craft. Manchester University Press, 1997.

[9] AATCC Committee RA89. (2012). AATCC Test Method 202-2012: Relative hand value of textiles: instrumental method. American Association of Textile Chemists and Colorists, p.404-406.

[10] M. Ashby, K. Johnson, Materials and Design: The art and science of material selection in product design. Butterworth-Heinemann, Oxford, 2010.

[11] E. Karana, O. Pedgley, \& V. Rognoli, Materials experience: fundamentals of materials and design. Elsevier, 2013.
[12] A. Light, Adding method to meaning: a technique for exploring peoples' experiences with technology. Beh. \& Information Technology, 2006, 25 (06): 175-187.

[13] D. Atkinson, P. Orzechowski, B. Petreca, et al., Tactile perceptions of digital textiles: a design research approach. CHI 2013, 1669-1678.

[14] C. Ho, R. Jones, S. King, L. Murray, \& C. Spence, Multisensory augmented reality in the context of a retail clothing application. In K. Bronner, R. Hirt, \& C. Ringe (Eds.), Audio Branding Academy yearbook 2012/2013, pp. 167-174, Germany: Nomos Publishers.

[15] B. Petreca, D. Atkinson, N. Bianchi-Berthouze, D. Furniss, S. Baurley, The future of textiles sourcing: exploring the potential for digital tools. Design \& Emotion International Conference, October 2014.

[16] S. Kawabata \& M. Nima, Fabric performance in clothing and clothing manufacture. J. of the Textile Institute, 1988, 80 (1), pp 19-50.

[17] B. Petreca, N. Bianchi-Berthouze, S. Baurley, P. Watkins, D. Atkinson, An embodiment perspective of affective touch behaviour In Experiencing Digital Textiles. ACII 2013.

[18] P. Dillon, W. Moody, R. Bartlett, P. Scully, R. Morgan, C. James, Sensing the fabric: To simulate sensation through sensory evaluation and in response to standard acceptable properties of specific materials when viewed as a digital image. Haptic HCI. Springer, 2000, p. 63-68.

[19] N. Magnenat-Thalmann, P. Volino, U. Bonanni, I. R. Summers, M. Bergamasco, F. Salsedo, F.E. Wolter, From physics-based simulation to the touching of textiles: The HAPTEX Project. The International Journal of Virtual Reality, 2007, 6 (3): 35-44.

[20] D. Atkinson, et al., Synthesising design methodologies for the transmission of tactile qualities in digital media. Digital Engagement 11 , November 15 - 17, 2011, Newcastle, UK.

[21] P.M. Orzechowski, "Interactive mobile presentation of textiles," Mobile HCI'10. ACM Press, 2010, pp.477-478.

[22] N. Bianchi-Berthouze, Understanding the role of body movement in player engagement. Human Computer Interaction, 2013.

[23] C. Petitmengin, A. Remillieux, B. Cahour, S. Carter-Thomas, A gap in Nisbett and Wilson's findings? A first-person access to our cognitive processes. Consciousness and Cognition, 2013, 22 (2), 654-669.

[24] P. Vermersch. L'entretien d'explicitation, ESF, 1994.

[25] F. Varela, \& J. Shear, First-person methodologies: What, Why, How? Journal of Consciousness Studies, 1999, 6, No. 2-3, pp. 1-14.

[26] M. Obrist, S.A. Seah, S. Subramanian, "Talking about tactile experiences," In CHI'13, pp.1659-1668.

[27] V. Braun, \& V. Clarke, Using thematic analysis in psychology. Qualitative Research in Psychology, 2006, 3(2), 77-101.

[28] G.K. Essick, F. McGlone, C. Dancer, D. Fabricant, Y. Raging, N. Phillips, T. Jones, S. Guest, Quantitative assessment of pleasant touch. Neuroscience and Biobehavioral Reviews, vol.34, 2010, pp.192-203.

[29] D. Kirsh, Embodied cognition and the magical future of interaction design. ACM Trans. Comput.-Hum. Interact, 2013, 20, 1, Article 3.

[30] A. Clark, Whatever next? Predictive brains, situated agents, and the future of cognitive science. Beh. and Brain Sciences, 2013, 36 (3): 1-24.

[31] A. Nöe, Action in perception. Cambridge: MIT Press, 2004.

[32] Obrist, M., Comber, R., Subramanian, S., Piqueras-Fiszman, B., Velasco, C., and Spence, C. (2014). Temporal, Affective, and Embodied Characteristics of Taste Experiences: A Framework for Design. CHI'14.

[33] Y. Gao, N. Bianchi-Berthouze, H. Meng, "What does touch tell us about emotions in touchscreen-based gameplay?" TOCHI, 2012.

[34] Petreca, B., Cary, L., Bianchi-Berthouze, N., Tajadura-Jiménez, A., Baurley, S. In our pockets: investigations of tactile experiences through touch behaviour. Poster presented at the First International Affective Touch Congress, 20-22 March 2015, UCL, London.

[35] Petreca, B. Prototyping tools to support the designer tactile experience with textiles. Report in preparation.

[36] D. Wu, T. Wu, H. Singh, S. Padilla, D. Atkinson, N. Bianchi-Berthouze M. Chantler, S. Baurley, "The Affective experience of handling digital fabrics: Tactile and visual cross-modal effects", Springer LNCS 6974, 2011, pp.427-436. 